\title{
Néo-mécanisme et approche informationnelle, culture et architecture numériques
}

\author{
Neo-mechanism and informational approach, digital culture \\ and architecture
}

Philippe Marin ${ }^{*}$

Univ. Grenoble Alpes, ENSAG, School of Architecture, MHA, 38000 Grenoble, France

\begin{abstract}
Résumé. Nous proposons de porter un regard sur la naissance des cultures numériques et sur l'usage de l'informatique comme outil d'aide à la conception. Notre objectif vise à donner une épaisseur historique aux cultures numériques, en les reliant à quelques moments remarquables de l'histoire, en les replaçant dans un contexte plus large. Ceci nous permet de contribuer à la compréhension des instrumentations numériques de l'architecture, à leurs acceptations et finalement à leurs appropriations créatives. Notre article est organisé en trois chapitres, nous considérons la naissance de l'automatisme du calcul, la naissance de l'ordinateur et l'émergence de l'architecture numérique. La généalogie que nous construisons nous donne à voir une trajectoire historique et contribue à penser, imaginer et concevoir nos outils contemporains.
\end{abstract}

Mots-clés. Culture numérique, culture matérielle, architecture numérique, histoire des techniques.

\begin{abstract}
We consider the digital culture emergence and the computer aided-design beginning. We aim to draw the history of this technical system, and to place its progress inside a broader context. In this way we would like to contribute to its understanding, to its acceptance, and eventually to its creative appropriation. We organize our discussion under three chapters: The birth of computation automatism, the birth of digital cultures, and the birth of digital architecture. The genealogy we build gives us a historical trajectory and contributes to think and imagine our contemporary tools.
\end{abstract}

Keywords. Digital culture, material culture, digital architecture, history of technology.

* philippe.marin@grenoble.archi.fr 


\section{Introduction}

Nous proposons de porter un regard sur la naissance des cultures numériques et sur l'usage de l'informatique comme outil d'aide à la conception. Notre objectif vise à relier les cultures numériques avec quelques moments remarquables de l'histoire. Notre regard n'est pas celui d'un historien, mais vise plutôt à la constitution d'un socle culturel, pour nous permettre de replacer le numérique dans une histoire longue, dans un contexte plus large, pour contribuer à sa compréhension, à son acceptation et finalement à son appropriation créative. Les relations que nous esquissons ici constituent nécessairement des raccourcis.

Nous organisons notre panorama autour de trois chapitres. Le premier s'intéresse à une période antérieure au XXe siècle, et il envisage l'automatisme du calcul comme un élément fondateur. Le second porte un regard sur la période qui voit naitre l'ordinateur, avec la formulation de la théorie de l'information et la naissance de la cybernétique. Nous envisageons la place de la contre-culture dans le phénomène d'appropriation de cet outil. Dans un troisième chapitre nous nous intéressons plus particulièrement à l'architecture numérique. Nous envisageons les premières formes de digitalisation de l'architecture, en considérant les démarches proto-paramétriques qui se prolongent aujourd'hui avec des méthodes de conception intégrée ou générative.

\section{Naissance de l'automatisme du calcul}

\subsection{La maitrise du temps et du mouvement}

Dans son séminaire doctoral sur la Naissance de la technologie (1970), Gilbert Simondon (1924-1989) développe l'idée que la technologie est déjà présente dans l'invention d'une machine simple. Celle-ci peut prendre la forme d'un outil, d'une roue, ou d'un faisceau de cordes. Ces éléments constituent des médiums entre l'opérateur et la matière naturelle, ils agissent les uns sur les autre dans une chaine d'opérations. Dans une machine plus complexe, cet enchainement des actions est rendu possible par l'alternance du rôle des éléments, passant successivement d'outil à opérateur, et constituant ainsi une séquence. Ce chainage renvoie à une forme d'automatisme qui est ainsi constitutif du machinisme [1]. Les questions de mécanisation, de régulation ou d'automatisation jalonnent l'histoire de l'humanité. De la clepsydre ou des horloges à eau, premiers instruments à mesurer le temps, aux métiers à tisser de Jacquard, première forme d'automatisation de la production, en passant par les automates à figure humaine ou animale, avec le Joueur de flûte ou le Canard de Jacques de Vaucanson (1709-1792) par exemple, l'histoire des techniques d'automatisation implique une maîtrise du temps et une maîtrise du mouvement. Un principe de régulation du mouvement peut être rendu possible par la pression hydraulique, le mouvement d'un pendule ou la pression d'un ressort. Une autre lignée de machines ordonne le mouvement à l'aide d'un « arbre à cames » dont l'agencement des différentes pièces permet de contrôler le mouvement de l'ensemble du système. Une illustration de ce dispositif se retrouve dans la boite à musique constituée d'un « cylindre à cames », celui-ci est hérissé de picots qui permettent la vibration de lames métalliques et ainsi la production d'une note de musique. Le changement du cylindre à cames reconfigure la machine et permet de jouer une autre mélodie. Le dispositif à cames, plus tard remplacé par les « cartons perforés », renvoie à un principe de programmation et d'automatisme de la machine. Cette programmation s'appuie sur un encodage binaire des mouvements [2]. 


\subsection{Les machines calculantes}

Parallèlement à cette culture matérielle, les techniques cognitives se constituent avec l'apparition des mathématiques, de l'alphabet et des écritures. Au IIXe siècle le mot algorithme apparait. Il correspond au patronyme latinisé d'Al-Khwarizmi, mathématicien, géographe, astrologue et astronome, né en Perse dans les années 780. Un autre personnage remarquable est Raymond Lulle (1232-1316). Il est philosophe, logicien et théologien, il est considéré comme un pionnier de la philosophie du numérique [3]. Il illustre une tentative d'algébrisation du langage et de la pensée en utilisant les méthodes logiques et la symbolique linguistique pour formaliser les raisonnements. Il consacra une partie de son œuvre à la réalisation d'une machine logique, instrument dénommé Ars magna, qui rassemblait un ensemble de prédicats, organisés à l'aide de diagrammes et de figures géométriques, et installés sur des cadrans mobiles. La machine était sensée conduire des raisonnements et démontrer la véracité des postulats. L'écossais John Napier (1550-1617), théologien, physicien, astronome et mathématicien, met au point un abaque, facilitant les opérations de multiplication et de division, appelé Bâton de Napier. La première machine à calculer est inventer par l'allemand Whihelm Schickard (1592-1635), elle porte le nom d'horloge calculante. Blaise Pascal (1623-1662) conçoit la Pascaline pour faciliter les fastidieux calcul de son père collecteur des impôts. Le philosophe et mathématicien allemand, Gottfried von Liebniz (1646-1716), construit une machine plus perfectionnée qui effectuait les quatre opérations. Plus tard, Charles Babbage (1791-1871), mathématicien et inventeur britannique, travaille également à la mise au point de machines composées de plusieurs manivelles, de pignons, de roues d'engrenages, dénommées machine à différence et machine analytique. Lady Lovelace (1815-1852), née Ada Byron, jeune fille douée en mathématique, compagne intellectuelle et disciple de Charles Babbage, travaille à la documentation et à l'amélioration de ces machines. Elle aurait largement contribué à l'écriture d'un algorithme de calcul, destiné à la machine de Babbage, et dont le formalisme se rapproche d'un véritable programme informatique [4]. Ces personnages illustrent les tentatives pour établir des relations entre le monde matériel et le monde de l'abstraction pure.

\subsection{Du calcul à l'encodage : naissance de l'informatique}

Cette ligné d'inventions peut se prolonger jusqu'à l'époque moderne. Avec les mathématiques et le télégraphe, l'idée de transfert de sens d'un niveau symbolique à un autre se propage, le principe de codage devient familier. Au XXe siècle l'idée que le langage du raisonnement pouvait être encodé se concrétise. Parallèlement, la naissance des états modernes est associée à des besoins d'inventaires, de recensements ou de productions de statistiques. Les périodes des deux guerres mondiales et de la guerre froide sont associées à des efforts en matière de recherche militaro-scientifique. Dans ce contexte de grands calculateurs sont mis au point à la fois pour tracer des tableaux de tirs de l'artillerie, des tables de positions de la lune, ou de traiter statistiquement de grandes masses de nombres [5]. L'automatisme, le calcul et l'information se trouvent mêlés pour donner naissance à une nouvelle lignée de machines basée sur une maîtrise du temps, du mouvement et des modalités de raisonnement. Les principes de régulation et de programmation se rencontrent pour façonner l'automatisation. Le terme informatique est créé par la réunion des termes information et automatique [2]. 


\section{Naissance de l'ordinateur}

\subsection{La théorie de l'information}

La théorie de l'information prend place dans le contexte du développement des communications à distance, avec le télégraphe aérien, le cryptage des messages, ou le télégraphe électrique. Les signaux électriques deviennent le support du message. En 1948, Claude Shannon (1916-2001) publie un article intitulé A Mathematical Theory of Communication. La théorie fournit une mesure de la quantité d'information contenue dans le choix élémentaire de deux possibilités et elle intègre une notion d'incertitude. L'encodage est réalisé de façon binaire, à l'aide de 0 et de 1 . Ce formalisme révèle toutes les capacités de la logique booléenne. L'information est à la fois une mesure et un symbole, l'informatique, en tant que traitement automatique, s'intéresse principalement au second sens (symbole numérique binaire). La théorie de l'information caractérise la capacité d'encodage des raisonnements et d'automatisation des calculs.

C'est notamment John von Neumann (1903-1957) qui établira les bases de la structure des grands calculateurs, ces derniers donneront à partir des années 60 naissance à l'informatique commerciale, aux premières utilisations de l'ordinateur à grande échelle, puis aux ordinateurs personnels et à la mise en réseau que nous connaissons aujourd'hui. John von Neumann, en tant que mathématicien et logicien, établira par ailleurs une « théorie des jeux », appliquée à différentes disciplines et proposant un formalisme mathématique à différents problèmes de stratégie. Alan Turing (1912-1954) s'intéressera à la notion de programmation de manière théorique et permettra une définition précise du concept d'algorithme. Il montrera le potentiel de l'algèbre comme support des raisonnements. Les opérations logiques se traduisent en opérations mathématiques. La manipulation formelle des concepts est remplacée par des opérations de nombres, les opérations conceptuelles sont traduites en opérations numériques.

\subsection{La cybernétique}

C'est dans ce contexte que le terme cybernétique voit le jour. Il est proposé par Norbert Wiener (1894-1964) à l'occasion de la parution de son ouvrage paru en 1948 Cybernetics : Or Control and Communication in the Animal and the Machine [6]. La méthode s'intéresse à la compréhension des phénomènes, à la fois naturels et artificiels, et passe par l'étude des processus de communication et de contrôle chez les êtres vivants et les machines. Pour Wiener, le processus de rétroaction représente le fondement de tout comportement organisé et intelligent. Il sert à décrire, dans un environnement en transformation, un dispositif informationnel qui assure à un phénomène un état d'équilibre ou le maintien de son action vers un but. La boucle de rétroaction, ou d'autorégulation, représente l'agent de stabilité d'un système. Des parallèles sont construits entre l'homme, l'animal, les phénomènes et la machine. Le traitement de l'information s'inscrit dans un cycle complet de perceptiondécision-action. Des analogies sont construites entre le cerveau, les modes de raisonnement et des machines câblées capables de reproduire certains comportements « intelligents ». Alan Turing publie son article intitulé Computing machinery and intelligence [7], il restera connu sous le nom de Test de Turing. Les premiers réseaux de neurones artificiels sont réalisés avec par exemple le modèle de perceptron de Frank Rosenblatt proposé en 1957. Après plusieurs cycles successifs de promesses et de désillusions, le domaine de l'intelligence artificielle retrouve une actualité dans les communautés scientifiques de cette première partie du XXIe siècle. 
Les interrelations, les boucles de rétroaction et l'échange d'informations contribuent à maintenir l'équilibre des systèmes ou leur orientation vers un but. Ces systèmes sont de différentes natures. Ils sont aussi bien des dispositifs fabriqués, des machines, qui vont du thermostat aux « animaux artificiels », que des systèmes vivants, à l'intérieur desquels des phénomènes biochimiques sont les éléments régulateurs.

L'approche cybernétique et la théorie de l'information donnent au concept d'information une dimension matérielle en le considérant comme un élément permettant d'organiser la matière, ou d'ordonner les phénomènes naturels, en compensant les processus entropiques. Ces acceptions ont contribué à envisager la transmission d'information et l'encodage comme le vecteur des interactions [8]. Après les machines constituées de roues, poulies et arbres à cames pour engendrer du mouvement et de l'énergie, apparaissent des machines électroniques, faites de transistors et de circuits intégrés, dans lesquelles le mouvement s'opère à l'échelle de la matière et entre les électrons. Ces machines sont programmées et reprogrammables jusqu'à présenter des formes d'autonomie à l'aide des boucles et processus récursifs sur lesquelles elles s'appuient. Un néo-mécanisme apparait à travers cette lignée de machines, avec la combinaison de la cybernétique et de l'informatique [9]. Depuis le début du XXIe siècle l'expression de système cyber-physique est utilisée pour qualifier les dispositifs composés de capteurs et d'actionneurs, capables tout à la fois de réaliser des mesures, de traiter des informations et d'opérer des actions dans le monde physique avec un certain degré d'autonomie.

\subsection{De la contre-culture à la cyberculture}

Parallèlement à ces développements scientifiques, un mouvement contribue à forger les cultures numériques, il est constitué par les communautés contre-culturelles américaines. Durant les années 1960-1970, de Sausalito à Drop City, elles seront nombreuses à s’installer dans l'ouest américain pour forger dans un esprit libertaire, des espaces d'expérimentation remettant en question les modes de vie, les systèmes productifs, proposant de nouvelles relations à la nature, explorant de nouveaux modes constructifs, remettant en question les systèmes hiérarchiques et l'organisation du pouvoir. Steve Baer (1938-) et son Dome Cookbook ou Ant Farm avec l'Inflatocookbook constituent une littérature de choix pour ces groupes de marginaux vivant en autonomie et convertis au «Do-It-Yourself » [10] [11]. Nous verrons au tournant du XXIe siècle une réactualisation de ces pratiques avec le développement des fab lab (laboratoire de fabrication numérique).

Au cœur de cet activisme, une autre publication se révèle un vecteur essentiel des cultures numériques. Il s'agit du Whole Earth Catalog, publié par Stewart Brand durant cette même période [12]. La publication prend la forme d'une encyclopédie de matériaux, un inventaire d'outils, une revue de littérature, un catalogue de vente par correspondance, un recueil de messages et de conseils. La revue est organisée en 9 chapitres dont les titres sont révélateurs des préoccupations : Understanding Whole Systems, Land Use, Shelter, Industry, Craft, Community, Nomadics, Communications, Learning. Au fil des numéros, et pour n'en citer que quelques-uns, les travaux des auteurs suivants se croisent : Richard Buckminster Fuller (1895-1983), Herbert Simon (1916-2001), Frei Otto (1925-2015), Victor Papanek (19231998), Norbert Wiener (1894-1964) ou encore Nicholas Negroponte (1943-). Les théories issues de la cybernétique croisent les propositions de Buckminster Fuller sur les systèmes dynamiques, la géométrie des cristaux et les formes fluides et organiques des systèmes naturels se combinent avec les diagrammes et les arborescences de la théorie de l'information et de la cybernétique. Le partage d'information et l'esprit collaboratif sont au cœur de ces activités.

Ainsi quatre groupes sociaux se croisent : les scientifiques et les technophiles, les scènes artistiques de New York et San Francisco, les communautés psychédéliques et les 
communautés intentionnelles ou alternatives [13] [14]. Au fil du temps, les partisans des technologies de pointe et ceux porteurs d'une philosophie orientale ou d'une théorie sociale communaliste, finissent par réunir les conditions culturelles pour faire de l'ordinateur et des réseaux informatiques des outils de libération. A travers ce dispositif sociotechnique, la perspective d'une société décentralisée, égalitaire, harmonieuse et libre, recueille tous les espoirs et toutes les attentions.

Parallèlement à ces pratiques culturelles, scientifiques ou militaires, une série de mises au point techniques vont progressivement rendre possible l'interconnexion des ordinateurs et donner naissance à l'internet. Nous mentionnons les progrès en matière de protocoles de communication entre les machines, avec la «commutation par parquets », les interfaces de consultation des données, avec le World Wild Web, ou encore les protocoles de communication entre individus, avec les mails ou les forums.

\section{4. Émergence de l'architecture numérique}

Nous souhaiterions dans cette dernière partie porter un regard plus spécifique sur les processus de conception architecturale. Cette brève histoire de l'architecture numérique porte sur une période qui débute avec l'antiquité et se déploie jusque dans les années 1960. Ce regard nous permet de dessiner une trajectoire qui va d'une pensée proto-paramétrique à l'apparition de l'interactivité que l'on trouve au sein des premières machines électroniques d'aide à la conception.

\subsection{D'une pensée proto-paramétrique au continuum conception-fabrication}

L'usage de l'encodage numérique, pour décrire des artefacts construits, trouve des origines lointaines. Mario Carpo explique ces pratiques par les contraintes de reproduction que les géographes et les architectes de l'antiquité ou de la renaissance rencontraient [15]. Il apparait plus facile de transmettre une suite de coordonnées, accompagnée des indications permettant l'élaboration du dessin et décrivant les opérations géométriques, que de réaliser des travaux de reproductions dont il était difficile d'assurer l'exactitude. C'est ce que Claude Ptolémée (100-168) réalisera avec son traité de géographie, intitulé Manuel pour dessiner une carte du monde, qui est considéré comme l'un des premiers atlas de la « totalité du monde connu », et qui prend la forme de tables de coordonnées accompagnées d'une méthode pour dessiner le monde habité. C'est également ce que Alberti (1404-1472) réalise avec sa publication Descriptio urbis Romae, parue dans les années 1440. Alberti décrit un système de coordonnées polaires et explicite comment dessiner le plan à partir de la liste des coordonnées rassemblées dans une table appelée Codex Chigi [16]. Mario Carpo suggère la parenté entre ces techniques de cartographie «à la demande» et les «affichages dynamiques » et personnalisés des néo-cartographies consultables aujourd'hui à l'aide de nos périphériques électroniques.

Cette approche numérique, pris au sens littéral et qui renvoie à l'usage des nombres, se retrouve également dans la conception des objets en trois dimensions. Dans l'histoire de l'art, nombreuses sont les compositions géométriques gouvernées par le nombre d'or. Ces approches s'appuient sur des principes récursifs et des logiques de séquençage. George Hersey [17] ou encore Park [18], ont montré comment mathématiser les villas de Palladio ou comment les entablements ioniques se décrivent à l'aide d'une succession d'instructions récursives.

Ces architectures de la renaissance révèlent des pensées et des processus protoparamétriques de conception et de fabrication. Leurs modes de description et de notation sont étroitement associés aux conditions de construction de l'époque qui les a vu naitre, ils 
facilitent des mises en œuvre sur chantier, impliquant les outils des tailleurs de pierre. Mais ils permettent également d'envisager ces archétypes comme des instances constituant des familles d'objets déclinables et que Mario Carpo rapproche de la notion «d'objectile» formulée par Deleuze à la fin des années 90 [19]. Avec le principe « d'objectile», les objets conçus et construits, s'inscrivent dans une forme de continuum, ils se constituent par la variation continue de leurs paramètres, et ils préfigurent les logiques de personnalisation de masse et d'architecture non standard qui émergent à la fin des années 90 avec les travaux de Bernard Cache [20] ou de Greg Lynn [21], [22] pour n'en citer que deux. Le continuum numérique conception-fabrication, constitue un principe de conception intégrée, rapprochant la modélisation et la fabrication en les intégrant dans un flux informationnel. Les modélisations intègrent les contraintes de fabrication, puis permettent la génération des programmes de commande des machines. Ces recherches deviendront progressivement capitales et constituent aujourd'hui des domaines de pointe. Elles questionnent les processus de fabrication, elles explorent des solutions constructives, elles redéfinissent les jeux d'acteurs, elles renouvellent les processus créatifs en rapprochant culture matérielle et culture numérique.

\subsection{Luigi Moretti et l'architecture paramétrique}

Le terme d'architettura parametrica apparait en 1960 à l'occasion de la 12ème Triennale de Milan où Luigi Moretti (1907-1973) présente ses travaux. Dès les années 1942, Luigi Moretti, accompagné par le mathématicien Bruno de Finetti, explorent l'usage des fonctions paramétriques pour la conception de formes construites. Ils définissent l'architecture paramétrique comme l'étude d'un système architecturale déterminé par les relations entre ses dimensions en fonctions de paramètres. Ils considèrent l'architecture comme une instance particulière dans un espace de solutions définies par l'ensemble des paramètres mobilisés. Ils feront un usage nouveau des techniques informatiques, mathématiques et statistiques en intégrant des logiques de variations [23]. Ils appliqueront notamment cette approche à la conception de stades sportifs pour chercher à optimiser la vue des spectateurs [24]. Cette exploration des techniques computationnelles sera appliquée à la distribution des chambres dans un complexe hôtelier, à la construction des cartes scolaires, à l'optimisation de la circulation routière ou encore à la planification urbaine. Luigi Moretti voyait dans l'usage des techniques informatiques et mathématiques un moyen d'objectivation et de formalisation d'intentions.

Cette période est associée à l'apparition des premiers calculateurs, et l'accès à ces ordinateurs va progressivement se généraliser et faciliter le recours aux formalismes mathématiques intégrant des séquences de calculs, des opérations logiques, des représentations graphiques électroniques, des sémantiques et des grammaires propres aux langages informatiques.

\subsection{Naissance des outils électroniques d'aide à la conception.}

Simultanément dans les laboratoires nord-américains, les premières recherches relatives à la mise au point de machines destinées à assister la conception se mettent en place. Le premier ordinateur équipé d'une interface de visualisation, nommé Whirlwind, voit le jour en 1951. Le projet $C A D$, acronyme de Computer-Aided Design, se développe de 1959 à 1970. Ces recherches sont conduites au sein de laboratoires d'ingénierie mécanique ou d'électronique, elles sont rendues possible par des programmes de financement proposés par l'armée, et trouvent des applications dans des domaines militaires ou dans les industries automobiles et aéronautiques [25] [5]. En France Pierre Bézier et Paul de Casteljau, respectivement ingénieur chez Renault et Citroën, proposent des solutions de notation 
paramétrique des formes libres et continues [26]. En 1963, Ivan Sutherland soutient sa thèse en présentant le projet Sketchpad, première interface de dessin en temps réel et qui préfigure nos environnements actuels de modélisation et de visualisation en ayant défini certains des concepts fondateurs de l'informatique, avec par exemple le fenestrage, les fonctionnalités de zoom ou la programmation par contraintes [25].

$\mathrm{Au}$ tournant des années 1970, Nicholas Negroponte et Leon Groisser fondent l'Architecture Machine Group au Massachussetts Institute of Technology (MIT), il évoluera en 1985 pour devenir le Media lab. Ce groupe de recherche applique ces nouveaux modes d'instrumentation à la conception architecturale. Ses membres envisagent le potentiel des outils informatiques, d'une part pour assister les processus de conception, en intégrant les progrès réalisés en sciences de la conception, avec l'émergence de l'intelligence artificielle, ou en envisageant des processus participatifs impliquant les usagers. Ils considèrent également l'ordinateur comme un moyen de reconfiguration de l'espace physique construit. Capacités de calcul et de simulation, concepts issus de la cybernétique, interfaces hommemachine, dispositifs d'interaction entre l'homme et les systèmes électroniques, ou modalités de transformation de l'espace bâti, sont au centre de ces recherches [27] [28].

Ces thématiques sont toujours d'actualité et les recherches sont nombreuse en matière d'interface homme-machine, d'instrumentation de l'espace et de la ville, d'analyse des processus de conception et des relations entre le concepteur et la machine, ou encore en matière d'intelligence artificielle et d'apprentissage automatique.

\section{Conclusion}

Cette brève lecture historique des évolutions, cette généalogie des techniques de conception, nous donne à voir son inscription temporelle, elle révèle son épaisseur historique en la reliant à une histoire longue de l'humanité et nous permet d'affirmer la prolongation de ses avancements. Nous avons relevé que plusieurs des thématiques actuelles de recherche trouvent leurs origines dans des travaux plus anciens. Nous retenons l'étroite relation entre culture matérielle et culture numérique, les liens entre activité de conception et activité de fabrication, ou encore la mathématisation et l'abstraction des activités cognitive pour constituer des outils d'aide à la conception.

Nous avons proposé à travers ces quelques lignes un panorama dans lequel se tisse des filiations entre les techniques, les sciences et les arts. Ces évolutions ont progressivement constitué un véritable système technique qui influence aujourd'hui nos activités cognitives et imprègne la société dans sa totalité. Cette généralisation est cependant soumise à de nombreuses critiques.

Nous pensons que l'acceptation des techniques de conception numérique est aujourd'hui moins confrontée à des verrous technologiques qu'à des freins culturels. Les mutations socioorganisationnelles qu'elles provoquent sont perçues comme des risques. Ainsi il nous semble que l'appropriations créatives des techniques numériques par les futurs concepteurs, passera notamment par la compréhension de leurs fonctionnements, et par la prise de connaissance de leurs origines et de leurs modes d'apparition.

La brève histoire de l'architecture numérique, décrite ici sur une période allant des années 1950 à 1970, fait l'objet de plusieurs publications et la littérature scientifique est nombreuse sur ce sujet. Celle-ci est cependant très largement analysée à travers une perspective anglosaxonne et nord-américaine. Il nous paraitrait nécessaire aujourd'hui de contribuer à la documentation de ce moment de l'histoire de l'informatique appliquée à l'architecture, en considérant plus spécifiquement les acteurs et les travaux réalisés dans l'hexagone ou au sein des communautés francophones. 


\section{Bibliographie}

[1] G. Simondon, Sur la technique (1953-1983), 1re édition. Paris: Presses universitaires de France, 2014.

[2] P. Breton, Une histoire de l'informatique. Seuil, 1990.

[3] W. Weibel, "Ramon Llull : Poet and pioneer of digital philosophy », in Dialogos Ramon Llulll's Méthod of thought and artistic practice, A. Vega, P. Weibel, et S. Zielinski, Éd. ZKM, 2018, p. 95-127.

[4] J. Gleick, L'information - L'histoire La théorie Le déluge, Cassini. 2015.

[5] D. C. Llach, Builders of the vision - Software and the imagination of design. Routledge, 2015.

[6] N. Wiener, La cybernétique information et régulation dans le vivant et la machine. Paris: Éd. du Seuil, 2014.

[7] A. M. Turing, "Computing machinery and intelligence », Mind, vol. LIX, $\mathrm{n}^{\circ}$ 236, p. 433-460, oct. 1950, doi: 10.1093/mind/LIX.236.433.

[8] M. Triclot, Le moment cybernétique : La constitution de la notion d'information. Editions Champ Vallon, 2008.

[9] G. Chazal, Philosophie de la machine: néo-mécanisme et post-humanisme. Dijon: Éditions Universitaires de Dijon, 2013.

[10] G. Borasi et M. Zardini, Désolé, plus d'essence: l'innovation architecturale en réponse à la crise pétrolière de 1973. Montréal: Centre Canadien d'Architecture, 2007.

[11] C. Maniaque Benton, Go west! des architectes au pays de la contre-culture. Marseille: Parenthèses, 2014.

[12] C. Maniaque Benton et M. Gaglio, Éd., Whole Earth field guide. Cambridge, Massachusetts: The MIT Press, 2016.

[13] D. Cardon, « Culture numérique ». 2019.

[14] F. Turner, Aux sources de l'utopie numérique: de la contre-culture à la cyberculture: Stewart Brand, un homme d'influence. Caen: C \& F, 2013.

[15] M. Carpo, The alphabet and the algorithm. Cambridge, Mass: MIT Press, 2011.

[16] B. Queysanne, "Les métiers de l'histoire de l'architecture. La description architecturale (I): Alberti et Raphaël "Descriptio Urbis Romae" », Ministère de l'équipement, du logement, de l'aménagement du territoire et des transports / Bureau de la recherche architecturale (BRA); Ministère de la recherche et de la technologie; Ecole nationale supérieure d'architecture de Grenoble / Les Métiers de l'Histoire de l'Architecture, Research Report 598/89, 1989. [En ligne]. Disponible sur: https://hal.archives-ouvertes.fr/hal-01906038.

[17] G. L. Hersey et R. Freedman, Possible Palladian villas: plus a few instructively impossible ones. Cambridge, Mass: MIT Press, 1992.

[18] H.-J. Park, « Parametric Variations of Palladio's Villa Rotonda », International Journal of Architectural Computing, vol. 6-n ${ }^{0}$ 2, p. 145-169, 2008.

[19] G. Deleuze, Le pli. Editions de Minuit, 1988.

[20] B. Cache, Terre meuble. Orléans: Editions HYX, 1997.

[21] G. Lynn, Animate form. New York: Princeton Architectural Press, 1999.

[22] G. Lynn, Éd., Folding in architecture, Rev. ed. Chichester, West Sussex ; Hoboken, NJ: Wiley-Academy, 2004.

[23] A. J. Wit, «L'animal machinique: les réseaux autonomes et la computation comportementale », in Quand le Numérique Marque-t-il l'Architecture?, A. Goodhouse, Éd. Centre Canadien d'Architecture, Sternberg Press, 2017, p. 215-278.

[24] R. Bottazzi, Digital architecture beyond computers: fragments of a cultural history of computational design. New York: Bloomsbury Visual Arts, An imprint of Bloomsbury Publishing Plc, 2018. 
[25] R. M. Correia et M. Guerreiro, « The Roots of 4IR in Architecture - A military drawing machine used for space perception in architecture », in Architecture in the Age of the 4th Industrial Revolution, Porto, Portugal, sept. 2019, vol. 1, p. 397-406.

[26] A. Townsend, "On the Spline: A Brief History of the Computational Curve », International journal of interior architecture + spatial design, vol. Issue 3, 2014.

[27] N. Negroponte, Soft architecture machines. Cambridge, Mass: The MIT Press, 1975.

[28] O. Halpern, «L'architecture comme machine : la ville intelligente déconstruite », in Quand le Numérique Marque-t-il l'Architecture?, A. Goodhouse, Éd. Centre Canadien d'Architecture, Sternberg Press, 2017, p. 464. 